\title{
Parametric Variogram Matrices Incorporating Both Bounded and Unbounded Functions
}

\author{
*Wanfang Chen · Marc G. Genton
}

Received: date / Accepted: date

\begin{abstract}
We construct a flexible class of parametric models for both traditional and pseudo variogram matrix (valued functions), where the off-diagonal elements are the traditional cross variograms and pseudo cross variograms, respectively, and the diagonal elements are the direct variograms, based on the method of latent dimensions and the linear model of coregionalization. The entries in the parametric variogram matrix allow for a smooth transition between boundedness and unboundedness by changing the values of parameters, and thus between joint second-order and intrinsically stationary vector random fields, or between multivariate geometric Gaussian processes and multivariate Brown-Resnick processes in spatial extreme analysis.
\end{abstract}

Keywords bounded and unbounded variogram · cross variogram · intrinsic stationarity $\cdot$ second-order stationarity $\cdot$ variogram matrix

\section{Introduction}

The variogram (Matheron 1963) is a function that quantifies the degree of spatial dependence of a univariate random field. The variogram of a univariate random field, $Z(s), s \in \mathbb{R}^{d}, d \geq 1$ is defined by the intrinsic stationarity

\footnotetext{
*Wanfang Chen

Statistics Program, King Abdullah University of Science and Technology, Thuwal 239556900, Saudi Arabia.

E-mail: wanfang.chen@kaust.edu.sa

Marc G. Genton

Statistics Program, King Abdullah University of Science and Technology, Thuwal 239556900, Saudi Arabia.
} 
hypothesis, which consists of two assumptions about the increments (Wackernagel 2003):

(i) the mean of the increments, called the drift, is invariant for any translation of a given vector $\boldsymbol{h} \in \mathbb{R}^{d}: \mathrm{E}\{Z(\boldsymbol{s}+\boldsymbol{h})-Z(\boldsymbol{s})\}=m(\boldsymbol{h})$ for all $\boldsymbol{s}, \boldsymbol{h} \in \mathbb{R}^{d}$;

(ii) the variance of the increments, $\operatorname{var}\{Z(\boldsymbol{s}+\boldsymbol{h})-Z(\boldsymbol{s})\}$, exists for all $\boldsymbol{s}, \boldsymbol{h} \in \mathbb{R}^{d}$ and is only a function of $\boldsymbol{h}$ for all $\boldsymbol{s}, \boldsymbol{h} \in \mathbb{R}^{d}$.

These two properties of an intrinsically stationary random function yield the definition for the (non-centered) theoretical variogram:

$$
\gamma(\boldsymbol{h})=\frac{1}{2} \operatorname{var}\{Z(\boldsymbol{s}+\boldsymbol{h})-Z(\boldsymbol{s})\}, \boldsymbol{s}, \boldsymbol{h} \in \mathbb{R}^{d} .
$$

In this paper, we focus on the case of random fields with no drift, i.e., $m(\boldsymbol{h})=0$, which implies that $\gamma(\boldsymbol{h})=\frac{1}{2} \mathrm{E}\left[\{Z(\boldsymbol{s}+\boldsymbol{h})-Z(\boldsymbol{s})\}^{2}\right]$. The existence of expectation and variance of the increments does not imply the existence of the first two moments of the random function itself: an intrinsic random function can have an infinite variance although the variance of its increments is finite for any vector $\boldsymbol{h}$. An intrinsically stationary random function does not need to have a constant mean or a constant variance. Hence, intrinsic stationarity is more general than second-order stationarity. By definition, the variogram has the following properties:

(a) the value of the variogram at the origin is zero: $\gamma(\mathbf{0})=0$;

(b) the values of the variogram are positive: $\gamma(\boldsymbol{h}) \geq 0$;

(c) the variogram is an even function: $\gamma(-\boldsymbol{h})=\gamma(\boldsymbol{h})$;

(d) the variogram has less than quadratic growth: $\lim _{\|\boldsymbol{h}\| \rightarrow \infty} \frac{\gamma(\boldsymbol{h})}{\|\boldsymbol{h}\|^{2}}=0$ (as otherwise the drift $m(\boldsymbol{h})$ could not be assumed zero);

(e) the variogram is conditionally negative definite: $\sum_{i=1}^{n} \sum_{j=1}^{n} a_{i} a_{j} \gamma\left(s_{i}-\right.$ $\left.\boldsymbol{s}_{j}\right) \leq 0$, for every integer $n \geq 2$ and any $a_{k} \in \mathbb{R}, k=1,2, \ldots, n$, such that $\sum_{k=1}^{n} a_{k}=0$.

The strict conditionally negative definiteness of the variogram is a sufficient condition for the system of linear equations for kriging to have a unique solution (Myers 1992). Note that although $\gamma(h)=1-\cos (h)$ is conditionally negative definite and bounded, it is not strictly conditionally negative definite.

A second-order stationary univariate random field can only be characterized by a bounded variogram, whereas the variogram of an intrinsically stationary random field might be unbounded. In spatial extreme value analysis, the Brown-Resnick processes on $\mathbb{R}^{d}$, originally introduced by Brown and Resnick 
(1977) and later extended by Kabluchko et al. (2009), constitute a flexible class of max-stable random fields that depends only on the variogram of an intrinsically stationary Gaussian process. The Brown-Resnick process is non-ergodic if the underlying variogram is bounded, while it is mixing if the variogram exhibits linear growth, and it is a mixed moving maximum process on the real line if the variogram $\gamma(h), h \in \mathbb{R}$, grows faster than $4 \log |h|$ (Kabluchko et al. 2009; Wang and Stoev 2010; Kabluchko and Schlather 2010). The Brown-Resnick processes are important in modeling extreme events of natural phenomena such as large floods, heat waves or windstorms. Non-ergodic models are suitable for modeling spatially extended events (e.g., cyclonic rainfall), whereas ergodicity can be assumed when the extremes are caused by local events (e.g., storms). Most variogram models form distinct classes that can only characterize either second-order stationarity or intrinsic stationarity of random fields (e.g., Cressie (1991), Chilès and Delfiner (2012), Wackernagel (2003), Porcu et al. (2007), Ma (2005, 2009) and Porcu and Schilling (2011)), or that can characterize either ergodic or non-ergodic Brown-Resnick processes (e.g., Thibaud et al. (2013) and Wadsworth and Tawn (2013)). When the kind of underlying causative process is unclear, a parametric model that allows for both bounded and unbounded variograms is advantageous. Recently, Schlather and Moreva (2017) proposed a simple model that allows for a smooth transition between a bounded and unbounded variogram:

$$
\gamma_{\alpha, \beta}(\boldsymbol{h})=\left\{\begin{array}{cl}
\frac{\left(1+\|\boldsymbol{h}\|^{\alpha}\right)^{\beta / \alpha}-1}{2^{\beta / \alpha}-1}, & \beta \neq 0 \\
\log \left(1+\|\boldsymbol{h}\|^{\alpha}\right) / \log 2, & \beta=0
\end{array}\right.
$$

where $\boldsymbol{h} \in \mathbb{R}^{d}$ is the lag vector between two locations, and $0<\alpha \leq 2,-\infty<$ $\beta<2$. The variogram $\gamma_{\alpha, \beta}$ is bounded if and only if $\beta<0$. The normalizing constant is chosen such that $\gamma_{\alpha, \beta}(1)=1$.

In this work, we aim at generalizing the univariate variogram model in Schlather and Moreva (2017) to variogram matrices for vector random fields, preserving the flexibility of varying between boundedness and unboundedness for the entries of the variogram matrix by changing the values of parameters in the model. Consider a $p$-variate random field $\boldsymbol{Z}(\boldsymbol{s})=\left\{Z_{1}(\boldsymbol{s}), \ldots, Z_{p}(\boldsymbol{s})\right\}^{T}$ defined on $\mathbb{R}^{d}$, where $Z_{i}(s), i=1, \ldots, p$ is the $i$ th component at location $s$. The direct and (traditional) cross variograms (Matheron 1965), $\gamma_{i j}(\boldsymbol{h})$, are defined for $p$ random functions as the natural generalization of the univariate 
variogram in the context of a joint intrinsic hypothesis (Wackernagel 2003):

$$
\begin{aligned}
& \mathrm{E}\left\{Z_{i}(\boldsymbol{s}+\boldsymbol{h})-Z_{i}(\boldsymbol{s})\right\}=0 \text { for all } \boldsymbol{s}, \boldsymbol{h} \in \mathbb{R}^{d} \text { and all } i=1, \ldots, p \\
& \operatorname{cov}\left\{Z_{i}(\boldsymbol{s}+\boldsymbol{h})-Z_{i}(\boldsymbol{s}), Z_{j}(\boldsymbol{s}+\boldsymbol{h})-Z_{j}(\boldsymbol{s})\right\}=2 \gamma_{i j}(\boldsymbol{h}) \\
& \quad \text { for all } \boldsymbol{s}, \boldsymbol{h} \in \mathbb{R}^{d} \text { and any pairs } i, j=1, \ldots, p .
\end{aligned}
$$

The direct and (traditional) cross variograms are thus defined as

$$
\gamma_{i j}(\boldsymbol{h})=\frac{1}{2} \mathrm{E}\left[\left\{Z_{i}(\boldsymbol{s}+\boldsymbol{h})-Z_{i}(\boldsymbol{s})\right\}\left\{Z_{j}(\boldsymbol{s}+\boldsymbol{h})-Z_{j}(\boldsymbol{s})\right\}\right], \boldsymbol{s}, \boldsymbol{h} \in \mathbb{R}^{d}, i, j=1, \ldots, p .
$$

These functions are even and they satisfy the inequalities

$$
\left|\gamma_{i j}(\boldsymbol{h})\right|^{2} \leq \gamma_{i i}(\boldsymbol{h}) \gamma_{j j}(\boldsymbol{h}), \quad \boldsymbol{h} \in \mathbb{R}^{d}, i, j=1, \ldots, p .
$$

Thus, according to the property (d) of a univariate variogram, the (traditional) cross variogram also has less than quadratic growth: $\lim _{\|\boldsymbol{h}\| \rightarrow \infty} \frac{\gamma_{i j}(\boldsymbol{h})}{\|\boldsymbol{h}\|^{2}}=0$.

A disadvantage of the above definition is that the measurements must be sited at the same geographical location, which leads to practical problems when attempting to estimate/model cross variograms, in particular the "undersampled" problem wherein there are locations with data values for only some of the components. This motivates another definition of cross variogram as proposed by Clark et al. (1989), and later generalized by Myers (1991) who coined it as the "pseudo cross variogram". According to Clark et al. (1989), the non-symmetric cross variograms assuming zero-mean random functions can be defined as

$$
\nu_{i j}(\boldsymbol{h})=\frac{1}{2} \mathrm{E}\left[\left\{Z_{i}(\boldsymbol{s}+\boldsymbol{h})-Z_{j}(\boldsymbol{s})\right\}^{2}\right], \quad \boldsymbol{s}, \boldsymbol{h} \in \mathbb{R}^{d}, i, j=1, \ldots, p .
$$

Myers (1991) observed that the equation in (4) are equal to the variances of the differences only if the random functions have constant and equal means. He therefore generalized the definition for the direct and pseudo cross variograms as

$$
\nu_{i j}(\boldsymbol{h})=\frac{1}{2} \operatorname{var}\left\{Z_{i}(\boldsymbol{s}+\boldsymbol{h})-Z_{j}(\boldsymbol{s})\right\}, \quad \boldsymbol{s}, \boldsymbol{h} \in \mathbb{R}^{d}, i, j=1, \ldots, p .
$$

All entries of the pseudo variogram matrix are nonnegative, while it may not be symmetric or positive definite.

Myers (1982) and Wackernagel (1988) have been early proponents of the cross variogram given by (2). Myers (1991) showed how cokriging equations could be written in terms of the pseudo cross variograms. The relationship 
and differences between these two definitions of cross variograms as well as the cokriging equations have been fully discussed in Myers (1991) and others (e.g., Cressie and Wikle (1998), Wackernagel (2003), Huang et al. (2009) and Genton and Kleiber (2015)).

Under joint second-order stationarity, that is, all the direct and cross covariance functions exist and depend on the spatial lag $\boldsymbol{h}$ only, the traditional variogram matrix and the cross covariance matrix are connected by (Wackernagel 2003)

$$
\gamma(\boldsymbol{h})=\mathbf{C}(\mathbf{0})-\frac{1}{2}\{\mathbf{C}(\boldsymbol{h})+\mathbf{C}(-\boldsymbol{h})\}, \boldsymbol{h} \in \mathbb{R}^{d},
$$

where $\gamma(\boldsymbol{h})=\left[\gamma_{i j}(\boldsymbol{h})\right], \mathbf{C}(\mathbf{0})=\left[C_{i j}(\mathbf{0})\right]=\left[\operatorname{cov}\left\{Z_{i}(\boldsymbol{s}), Z_{j}(\boldsymbol{s})\right\}\right]$ and $\mathbf{C}(\boldsymbol{h})=$ $\left[C_{i j}(\boldsymbol{h})\right]=\left[\operatorname{cov}\left\{Z_{i}(\boldsymbol{s}+\boldsymbol{h}), Z_{j}(\boldsymbol{s})\right\}\right]$. Thus, under joint second-order stationarity, the entries in the traditional variogram matrix are all bounded due to the Cauchy-Schwarz inequality:

$$
\left|C_{i j}(\boldsymbol{h})\right|^{2} \leq C_{i i}(\mathbf{0}) C_{j j}(\mathbf{0}), \boldsymbol{h} \in \mathbb{R}^{d}, i, j=1, \ldots, p
$$

Similarly, the entries in a pseudo variogram matrix are all bounded under joint second-order stationarity due to the relationship (Wackernagel 2003):

$$
\nu_{i j}(\boldsymbol{h})=\frac{1}{2}\left\{C_{i i}(\mathbf{0})+C_{j j}(\mathbf{0})\right\}-C_{i j}(\boldsymbol{h}), \boldsymbol{h} \in \mathbb{R}^{d}, i, j=1, \ldots, p .
$$

Under joint intrinsic stationarity, however, the entries in the variogram matrices might be unbounded. In the multivariate max-stable spatial processes (Genton et al. 2015b), the multivariate Brown-Resnick process (Molchanov and Stucki 2013) is the multivariate geometric Gaussian process (Davison and Gholamrezaee 2012) if it is joint second-order stationary. Thus, a variogram matrix model with the direct and cross variograms that incorporate both boundedness and unboundedness can be advantageous, for example, if it is unclear whether the multivariate max-stable process is a multivariate geometric Gaussian or a Brown-Resnick process.

Both types of variogram matrix must satisfy the inequality

$$
\sum_{i=1}^{n} \sum_{j=1}^{n} \boldsymbol{a}_{i}^{T} \gamma\left(\boldsymbol{s}_{i}, \boldsymbol{s}_{j}\right) \boldsymbol{a}_{j} \leq 0,
$$

for every integer $n \geq 2$ and any $\boldsymbol{a}_{k} \in \mathbb{R}^{p}, k=1,2, \ldots, n$, such that $\sum_{k=1}^{n} \boldsymbol{a}_{k}=$ 0 (Ma 2011b). This reduces to the conditionally negative definite condition for a univariate variogram when $p=1$. 
It is often nontrivial to verify the inequality (6). Based on latent dimensions (Apanasovich and Genton 2010), an approach to constructing a traditional variogram matrix from existing univariate variograms was put forward by $\mathrm{Ma}$ (2011a). The key idea of latent dimensions is to represent components of a vector in $\mathbb{R}^{d}$ as points in $\mathbb{R}^{q}(1 \leq q \leq p$, where $p$ is the length of the vector). Then, based on these latent dimensions, the direct and cross variograms become functions with arguments from a higher dimension, $\mathbb{R}^{d+q}$. Specifically, suppose each of the components of a $p$-variate random field $\boldsymbol{Z}(\boldsymbol{s})=\left\{Z_{1}(\boldsymbol{s}), \ldots, Z_{p}(\boldsymbol{s})\right\}^{T}$, $s \in \mathbb{R}^{d}$, can be represented as

$$
Z_{i}(\boldsymbol{s})=Z_{0}\left(\boldsymbol{s} ; \boldsymbol{\theta}_{i}\right), i=1,2, \ldots, p,
$$

where $\left\{Z_{0}(\boldsymbol{s} ; \boldsymbol{\theta}), \boldsymbol{s} \in \mathbb{R}^{d}, \boldsymbol{\theta} \in \mathbb{R}^{q}\right\}$ is a univariate random field with variogram $\gamma_{0}$. Then, the direct and cross variograms can be written as a linear combination of the $\gamma_{0} \mathrm{~s}$. Ma (2011a) used this approach to construct a class of (traditional) variogram matrices (see his Theorems 4 and 5) based on existing univariate variograms proposed by Ma (2005).

In this paper, we propose a class of valid parametric variogram matrices, both traditional and pseudo, that is a combination and extension of the main results from Ma (2011a) and Schlather and Moreva (2017), which are connected by the Bernstein function. The entries of this new class of variogram matrices allow for both bounded and unbounded variograms or cross variograms by changing the parameters in each entry independently.

The remainder of this paper is organized as follows. Sections 2 and 3 present the parametric traditional and pseudo variogram matrix, respectively. In Section 4 , the variogram matrix models for a bivariate random field are presented as a special case, with a visuanimation (Genton et al. 2015a) in the electronic supplementary material showing the transition between the bounded and unbounded direct and cross variograms. In Section 5, we propose a method for building parametric variogram matrice with different diagonal entries based on the Linear Model of Coregionalization (LMC), in order to overcome a limitation of the previous models. Section 6 gives a discussion, in which the main results of this study are summarized and both challenges and future works are discussed.

\section{Parametric Traditional Variogram Matrix Models}

In this section, we construct two types of valid traditional variogram matrix with their entries varying between boundedness and unboundedness. 
Consider a joint intrinsically stationary $p$-variate random field $\boldsymbol{Z}(\boldsymbol{s})=$ $\left\{Z_{1}(s), \ldots, Z_{p}(s)\right\}^{T}$ defined on $\mathbb{R}^{d}$, where $Z_{i}(s), i=1, \ldots, p$ is the $i$ th component at location $s$. The $p \times p$ traditional variogram matrix function is

$$
\gamma(\boldsymbol{h})=\left(\begin{array}{cccc}
\gamma_{11}(\boldsymbol{h}) & \gamma_{12}(\boldsymbol{h}) & \cdots & \gamma_{1 p}(\boldsymbol{h}) \\
\gamma_{21}(\boldsymbol{h}) & \gamma_{22}(\boldsymbol{h}) & \cdots & \gamma_{2 p}(\boldsymbol{h}) \\
\vdots & \vdots & \ddots & \vdots \\
\gamma_{p 1}(\boldsymbol{h}) & \gamma_{p 2}(\boldsymbol{h}) & \cdots & \gamma_{p p}(\boldsymbol{h})
\end{array}\right)
$$

where $\boldsymbol{h}=\boldsymbol{s}_{1}-\boldsymbol{s}_{2} \in \mathbb{R}^{d}$. The diagonal entries are direct variograms and the off-diagonal entries are the traditional cross variograms defined in (2).

Next, since any continuous, isotropic univariate variogram $\gamma(\boldsymbol{h})$ in $\mathbb{R}^{d}$ can be written in terms of a Bernstein function (Schoenberg 1938; Bochner 2005):

$$
\gamma(\boldsymbol{h})=B\left(\|\boldsymbol{h}\|^{2}\right), \boldsymbol{h} \in \mathbb{R}^{d}
$$

where $B(\cdot)$ is a Bernstein function on $[0, \infty)$ with $B(0)=0$, we choose the variogram model in our Equation (1) from Schlather and Moreva (2017) as the Bernstein function in Theorem 4 of Ma (2011a) (i.e., $B\left(\|\boldsymbol{h}\|^{2}\right)=\gamma_{\alpha, \beta}(\boldsymbol{h})$ ). The traditional variogram matrix $\gamma(\boldsymbol{h})$ is then formulated as follows.

Model 1 Suppose that $0<\alpha \leq 2,-\infty<\beta<2,0<\alpha_{1}<\alpha_{2}$, and $\theta_{i j}=\left\|\boldsymbol{\theta}_{i}-\boldsymbol{\theta}_{j}\right\|^{2}$, where $\boldsymbol{\theta}_{i}, \boldsymbol{\theta}_{j} \in \mathbb{R}^{q}, i, j=1, \ldots, p$.

(i) If $\left\{1-\left(\alpha_{2} / \alpha_{1}\right)^{(d+p) / 2}\right\}^{-1} \leq \lambda \leq 1$, then a matrix with the following entries:

$$
\begin{aligned}
\gamma_{i j}(\boldsymbol{h})=\left(2^{\beta / \alpha}-1\right)^{-1} & \left\{\lambda\left(\left[1+\left\{\alpha_{1}\left(\|\boldsymbol{h}\|^{2}+\theta_{i j}\right)\right\}^{\alpha / 2}\right]^{\beta / \alpha}-\left\{1+\left(\alpha_{1} \theta_{i j}\right)^{\alpha / 2}\right\}^{\beta / \alpha}\right)\right. \\
+ & \left.(1-\lambda)\left(\left[1+\left\{\alpha_{2}\left(\|\boldsymbol{h}\|^{2}+\theta_{i j}\right)\right\}^{\alpha / 2}\right]^{\beta / \alpha}-\left\{1+\left(\alpha_{2} \theta_{i j}\right)^{\alpha / 2}\right\}^{\beta / \alpha}\right)\right\}, \\
\boldsymbol{h} & \in \mathbb{R}^{d}, i, j=1, \ldots, p,
\end{aligned}
$$

where $\beta \neq 0$, is a valid traditional variogram matrix for a $p$-variate random field in $\mathbb{R}^{d}$. Since $\theta_{i i}=0, i=1, \ldots, p$, all the diagonal elements of $\gamma(\boldsymbol{h})$ are 
the same: $\gamma_{11}(\boldsymbol{h})=\cdots=\gamma_{p p}(\boldsymbol{h})$. When $\beta \rightarrow 0$, the limiting function is

$$
\begin{aligned}
\gamma_{i j}(\boldsymbol{h})=\{\log (2)\}^{-1} & \left\{\lambda\left(\log \left[1+\left\{\alpha_{1}\left(\|\boldsymbol{h}\|^{2}+\theta_{i j}\right)\right\}^{\alpha / 2}\right]-\log \left\{1+\left(\alpha_{1} \theta_{i j}\right)^{\alpha / 2}\right\}\right)\right. \\
& \left.+(1-\lambda)\left(\log \left[1+\left\{\alpha_{2}\left(\|\boldsymbol{h}\|^{2}+\theta_{i j}\right)\right\}^{\alpha / 2}\right]-\log \left\{1+\left(\alpha_{2} \theta_{i j}\right)^{\alpha / 2}\right\}\right)\right\}, \\
& \boldsymbol{h} \in \mathbb{R}^{d}, i, j=1, \ldots, p .
\end{aligned}
$$

If $\lambda \in[0,1]$, then all the direct and cross variograms in the traditional variogram matrix $\gamma(\boldsymbol{h})$ are univariate variograms.

(ii) If $\left\{1-\left(\alpha_{2} / \alpha_{1}\right)^{d+p}\right\}^{-1} \leq \lambda \leq\left(1-\alpha_{1} / \alpha_{2}\right)^{-1}$, then the traditional variogram matrix model for a $p$-variate random field defined in $\mathbb{R}^{d}$ is the same as in (i) except that the power of $\theta_{i j}$ and $\|\boldsymbol{h}\|^{2}+\theta_{i j}$ in the formula is $\alpha / 4$ instead of $\alpha / 2$.

According to Theorem 4 of Ma (2011a), Model 1 is a valid class of variogram matrices for vector random fields because we choose a specific Bernstein function $B(\cdot)$. Also, since $B(\cdot)$ is the variogram model in Schlather and Moreva (2017), the entries in the variogram matrix can vary between boundedness and unboundedness. Specifically, the direct and cross variograms $\gamma_{i j}(\boldsymbol{h}), i, j=1, \ldots, p$ are bounded if and only if $\beta<0$.

If we choose the Bernstein functions from Theorem 5 of Ma (2011a) as $B_{1}\left(\|\boldsymbol{h}\|^{2}\right)=\gamma_{\alpha_{1}, \beta_{1}}(\boldsymbol{h})$ and $B_{2}\left(\|\boldsymbol{h}\|^{2}\right)=\gamma_{\alpha_{2}, \beta_{2}}(\boldsymbol{h})$, where $0<\alpha_{1}, \alpha_{2}<2,-\infty<$ $\beta_{1}, \beta_{2}<2$, then we can formulate another class of valid traditional variogram matrix as follows.

Model 2 Suppose that $0<\alpha_{1}, \alpha_{2} \leq 2,-\infty<\beta_{1}<2,-\infty<\beta_{2}<2$, and $\theta_{i j}=\left\|\boldsymbol{\theta}_{i}-\boldsymbol{\theta}_{j}\right\|^{2}$, where $\boldsymbol{\theta}_{i}, \boldsymbol{\theta}_{j} \in \mathbb{R}^{q}, i, j=1, \ldots, p$.

(i) If $\beta_{1} \neq 0, \beta_{2} \neq 0$, then a matrix with the following entries:

$$
\begin{aligned}
\gamma_{i j}(\boldsymbol{h})= & \left(\left[\left\{1+\left(\|\boldsymbol{h}\|^{2}+\theta_{i j}\right)^{\alpha_{1} / 4}\right\}^{\beta_{1} / \alpha_{1}}-1\right]\left[\left\{1+\left(\|\boldsymbol{h}\|^{2}+\theta_{i j}\right)^{\alpha_{2} / 4}\right\}^{\beta_{2} / \alpha_{2}}-1\right]\right. \\
& \left.-\left\{\left(1+\theta_{i j}^{\alpha_{1} / 4}\right)^{\beta_{1} / \alpha_{1}}-1\right\}\left\{\left(1+\theta_{i j}^{\alpha_{2} / 4}\right)^{\beta_{2} / \alpha_{2}}-1\right\}\right) /\left\{\left(2^{\beta_{1} / \alpha_{1}}-1\right)\left(2^{\beta_{2} / \alpha_{2}}-1\right)\right\}, \\
& \boldsymbol{h} \in \mathbb{R}^{d}, i, j=1, \ldots, p,
\end{aligned}
$$

is a valid traditional variogram matrix for a $p$-variate random field defined in $\mathbb{R}^{d}$. Again, all the diagonal entries of the variogram matrix $\gamma(\boldsymbol{h})$ are the same. 
(ii) If $\beta_{1} \rightarrow 0, \beta_{2} \neq 0$, then

$$
\begin{aligned}
\gamma_{i j}(\boldsymbol{h})= & \left(\log \left\{1+\left(\|\boldsymbol{h}\|^{2}+\theta_{i j}\right)^{\alpha_{1} / 4}\right\}\left[\left\{1+\left(\|\boldsymbol{h}\|^{2}+\theta_{i j}\right)^{\alpha_{2} / 4}\right\}^{\beta_{2} / \alpha_{2}}-1\right]\right. \\
& \left.-\log \left(1+\theta_{i j}^{\alpha_{1} / 4}\right)\left\{\left(1+\theta_{i j}^{\alpha_{2} / 4}\right)^{\beta_{2} / \alpha_{2}}-1\right\}\right) /\left\{\left(2^{\beta_{2} / \alpha_{2}}-1\right) \log (2)\right\}, \\
& \boldsymbol{h} \in \mathbb{R}^{d}, i, j=1, \ldots, p .
\end{aligned}
$$

(iii) If $\beta_{2} \rightarrow 0, \beta_{1} \neq 0$, then

$$
\begin{aligned}
\gamma_{i j}(\boldsymbol{h})= & \left(\left[\left\{1+\left(\|\boldsymbol{h}\|^{2}+\theta_{i j}\right)^{\alpha_{1} / 4}\right\}^{\beta_{1} / \alpha_{1}}-1\right] \log \left\{1+\left(\|\boldsymbol{h}\|^{2}+\theta_{i j}\right)^{\alpha_{2} / 4}\right\}\right. \\
& \left.-\left\{\left(1+\theta_{i j}^{\alpha_{1} / 4}\right)^{\beta_{1} / \alpha_{1}}-1\right\} \log \left(1+\theta_{i j}^{\alpha_{2} / 4}\right)\right) /\left\{\left(2^{\beta_{1} / \alpha_{1}}-1\right) \log (2)\right\}, \\
& \boldsymbol{h} \in \mathbb{R}^{d}, i, j=1, \ldots, p .
\end{aligned}
$$

(iv) If $\beta_{1} \rightarrow 0, \beta_{2} \rightarrow 0$, then

$$
\begin{aligned}
\gamma_{i j}(\boldsymbol{h})= & {\left[\log \left\{1+\left(\|\boldsymbol{h}\|^{2}+\theta_{i j}\right)^{\alpha_{1} / 4}\right\} \log \left\{1+\left(\|\boldsymbol{h}\|^{2}+\theta_{i j}\right)^{\alpha_{2} / 4}\right\}\right.} \\
& \left.-\log \left(1+\theta_{i j}^{\alpha_{1} / 4}\right) \log \left(1+\theta_{i j}^{\alpha_{2} / 4}\right)\right] /\{\log (2)\}^{2} \\
& \boldsymbol{h} \in \mathbb{R}^{d}, i, j=1, \ldots, p .
\end{aligned}
$$

According to Theorem 5 of Ma (2011a), Model 2 is a valid class of variogram matrices for vector random fields because we choose specific Bernstein functions $B_{1}(\cdot)$ and $B_{2}(\cdot)$. Also, since $B_{1}(\cdot)$ and $B_{2}(\cdot)$ are chosen to be the variogram model in Schlather and Moreva (2017), the entries in the variogram matrix can vary between boundedness and unboundedness. Specifically, the direct and cross variograms $\gamma_{i j}(\boldsymbol{h}), i, j=1, \ldots, p$ are bounded if and only if $\beta_{1}<0$ and $\beta_{2}<0$.

\section{Parametric Pseudo Variogram Matrix Models}

In this section, we propose a class of valid pseudo variogram matrix that are based on latent dimensions and allow for boundedness and unboundedness in the entries of the matrix. 
Consider the pseudo variogram matrix for a joint intrinsically stationary $p$-variate random field $\boldsymbol{Z}(\boldsymbol{s})=\left\{Z_{1}(\boldsymbol{s}), \ldots, Z_{p}(\boldsymbol{s})\right\}^{T}, \boldsymbol{s} \in \mathbb{R}^{d}$ :

$$
\boldsymbol{\nu}(\boldsymbol{h})=\left(\begin{array}{cccc}
\nu_{11}(\boldsymbol{h}) & \nu_{12}(\boldsymbol{h}) & \cdots & \nu_{1 p}(\boldsymbol{h}) \\
\nu_{21}(\boldsymbol{h}) & \nu_{22}(\boldsymbol{h}) & \cdots & \nu_{2 p}(\boldsymbol{h}) \\
\vdots & \vdots & \ddots & \vdots \\
\nu_{p 1}(\boldsymbol{h}) & \nu_{p 2}(\boldsymbol{h}) & \cdots & \nu_{p p}(\boldsymbol{h})
\end{array}\right),
$$

where $\boldsymbol{h}=\boldsymbol{s}_{1}-\boldsymbol{s}_{2} \in \mathbb{R}^{d}$. The diagonal entries are direct variograms and the off-diagonal entries are the pseudo cross variograms defined in (4).

Following the idea of latent dimensions (Apanasovich and Genton 2010; Ma 2011a), we suppose that

$$
Z_{i}(\boldsymbol{s})=Z_{0}\left(\boldsymbol{s} ; \boldsymbol{\theta}_{i}\right), i=1,2, \ldots, p,
$$

where $\left\{Z_{0}(\boldsymbol{s} ; \boldsymbol{\theta}), \boldsymbol{s} \in \mathbb{R}^{d}, \boldsymbol{\theta} \in \mathbb{R}^{q}\right\}$ is a univariate random field with variogram $\gamma_{0}$. Then,

$$
\nu_{i j}(\boldsymbol{h})=\frac{1}{2} \operatorname{var}\left\{Z_{0}\left(\boldsymbol{s}+\boldsymbol{h} ; \boldsymbol{\theta}_{i}\right)-Z_{0}\left(\boldsymbol{s} ; \boldsymbol{\theta}_{j}\right)\right\}=\gamma_{0}\left(\boldsymbol{h} ; \boldsymbol{\theta}_{i}, \boldsymbol{\theta}_{j}\right) .
$$

Hence, a pseudo variogram matrix can be formed by directly inserting univariate variograms into its entries. For example, if we use the valid univariate variograms proposed by Ma (2005), where the Bernstein functions are chosen in the same way as described in Section 2, then we can formulate a class of pseudo variogram matrix with entries that brigde between bounded and unbounded direct or cross variograms. Below, Model 3 is a class of pseudo variogram matrix based on linear combinations of Bernstein functions, and Model 4 is another class of pseudo variogram matrix based on the product of Bernstein functions.

Model 3 Suppose that $0<\alpha_{1}<\alpha_{2}, 0<\alpha \leq 2,-\infty<\beta<2$, and $\theta_{i j}=\left\|\boldsymbol{\theta}_{i}-\boldsymbol{\theta}_{j}\right\|^{2}$, where $\boldsymbol{\theta}_{i}, \boldsymbol{\theta}_{j} \in \mathbb{R}^{q}, i, j=1, \ldots, p$.

(i) If $\left\{1-\left(\alpha_{2} / \alpha_{1}\right)^{(d+p) / 2}\right\}^{-1} \leq \lambda \leq 1$, then a matrix with the following entries:

$$
\begin{aligned}
\nu_{i j}(\boldsymbol{h})= & \left\{\lambda\left(\left[1+\left\{\alpha_{1}\left(\|\boldsymbol{h}\|^{2}+\theta_{i j}\right)\right\}^{\alpha / 2}\right]^{\beta / \alpha}-1\right)\right. \\
& \left.+(1-\lambda)\left(\left[1+\left\{\alpha_{2}\left(\|\boldsymbol{h}\|^{2}+\theta_{i j}\right)\right\}^{\alpha / 2}\right]^{\beta / \alpha}-1\right)\right\} /\left(2^{\beta / \alpha}-1\right), \\
& \boldsymbol{h} \in \mathbb{R}^{d}, i, j=1, \ldots, p
\end{aligned}
$$


where $\boldsymbol{h}=\boldsymbol{s}_{1}-\boldsymbol{s}_{2}$ and $\beta \neq 0$, is a valid pseudo variogram matrix for a $p$-variate random field defined in $\mathbb{R}^{d}$. Again, since $\theta_{i i}=0, i=1, \ldots, p$, all the diagonal entries of the variogram matrix $\boldsymbol{\nu}(\boldsymbol{h})$ are the same. When $\beta \rightarrow 0$, the limiting function is

$$
\begin{aligned}
\nu_{i j}(\boldsymbol{h})= & \left\{\lambda \log \left[1+\left\{\alpha_{1}\left(\|\boldsymbol{h}\|^{2}+\theta_{i j}\right)\right\}^{\alpha / 2}\right]+(1-\lambda) \log \left[1+\left\{\alpha_{2}\left(\|\boldsymbol{h}\|^{2}+\theta_{i j}\right)\right\}^{\alpha / 2}\right]\right\} / \log (2), \\
& \boldsymbol{h} \in \mathbb{R}^{d}, i, j=1, \ldots, p,
\end{aligned}
$$

(ii) If $\left\{1-\left(\alpha_{2} / \alpha_{1}\right)^{d+p}\right\}^{-1} \leq \lambda \leq\left(1-\alpha_{1} / \alpha_{2}\right)^{-1}$, then the pseudo variogram matrix model for a $p$-variate random field defined in $\mathbb{R}^{d}$ is the same as in (i) except that the power of $\|\boldsymbol{h}\|^{2}+\theta_{i j}$ in the formula is $\alpha / 4$ instead of $\alpha / 2$.

Model 4 Suppose that $0<\alpha_{1}, \alpha_{2} \leq 2,-\infty<\beta_{1}<2,-\infty<\beta_{2}<2$, and $\theta_{i j}=\left\|\boldsymbol{\theta}_{i}-\boldsymbol{\theta}_{j}\right\|^{2}$, where $\boldsymbol{\theta}_{i}, \boldsymbol{\theta}_{j} \in \mathbb{R}^{q}, i, j=1, \ldots, p$.

(i) If $\beta_{1} \neq 0, \beta_{2} \neq 0$, then a matrix with the following entries:

$$
\begin{aligned}
\nu_{i j}(\boldsymbol{h})= & \left\{\left[\left\{1+\left(\|\boldsymbol{h}\|^{2}+\theta_{i j}\right)^{\alpha_{1} / 4}\right\}^{\beta_{1} / \alpha_{1}}-1\right]\left[\left\{1+\left(\|\boldsymbol{h}\|^{2}+\theta_{i j}\right)^{\alpha_{2} / 4}\right\}^{\beta_{2} / \alpha_{2}}-1\right]\right\} \\
& /\left\{\left(2^{\beta_{1} / \alpha_{1}}-1\right)\left(2^{\beta_{2} / \alpha_{2}}-1\right)\right\}, \\
& \boldsymbol{h} \in \mathbb{R}^{d}, i, j=1, \ldots, p,
\end{aligned}
$$

is a valid pseudo variogram matrix for a $p$-variate random field defined in $\mathbb{R}^{d}$. Again, all the diagonal entries of the variogram matrix $\boldsymbol{\nu}(\boldsymbol{h})$ are the same.

(ii) If $\beta_{1} \rightarrow 0, \beta_{2} \neq 0$, then

$$
\begin{aligned}
\nu_{i j}(\boldsymbol{h})= & \left\{\log \left[1+\left(\|\boldsymbol{h}\|^{2}+\theta_{i j}\right)^{\alpha_{1} / 4}\right]\left[\left\{1+\left(\|\boldsymbol{h}\|^{2}+\theta_{i j}\right)^{\alpha_{2} / 4}\right\}^{\beta_{2} / \alpha_{2}}-1\right]\right\} \\
& /\left\{\left(2^{\beta_{2} / \alpha_{2}}-1\right) \log (2)\right\}, \\
& \boldsymbol{h} \in \mathbb{R}^{d}, i, j=1, \ldots, p .
\end{aligned}
$$

(iii) If $\beta_{2} \rightarrow 0, \beta_{1} \neq 0$, then

$$
\begin{aligned}
\nu_{i j}(\boldsymbol{h})= & \left\{\left[\left\{1+\left(\|\boldsymbol{h}\|^{2}+\theta_{i j}\right)^{\alpha_{1} / 4}\right\}^{\beta_{1} / \alpha_{1}}-1\right] \log \left[1+\left(\|\boldsymbol{h}\|^{2}+\theta_{i j}\right)^{\alpha_{2} / 4}\right]\right\} \\
& /\left\{\left(2^{\beta_{1} / \alpha_{1}}-1\right) \log (2)\right\} \\
& \boldsymbol{h} \in \mathbb{R}^{d}, i, j=1, \ldots, p .
\end{aligned}
$$


(iv) If $\beta_{1} \rightarrow 0, \beta_{2} \rightarrow 0$, then

$$
\begin{aligned}
\nu_{i j}(\boldsymbol{h})= & \left\{\log \left[1+\left(\|\boldsymbol{h}\|^{2}+\theta_{i j}\right)^{\alpha_{1} / 4}\right] \log \left[1+\left(\|\boldsymbol{h}\|^{2}+\theta_{i j}\right)^{\alpha_{2} / 4}\right]\right\} /\{\log (2)\}^{2}, \\
& \boldsymbol{h} \in \mathbb{R}^{d}, i, j=1, \ldots, p .
\end{aligned}
$$

Similar to Section 2, these models are valid pseudo variogram matrices. Interestingly, the differences between the corresponding entries in the proposed traditional and pseudo variogram matrix are constant with respect to $\boldsymbol{h}$ for any fixed combinations of parameters, whether we compare Model 1 to Model 3, or Model 2 to Model 4.

\section{The Bivariate Case}

To better understand how the variogram matrices proposed in Sections 2 and 3 allow for both bounded and unbounded direct variograms or cross variograms in their entries, we consider the bivariate case (i.e., $p=2$ ). It is straightforward to see that both the traditional and pseudo variogram matrices are symmetric, and they share the same diagonal entries, which are the direct variograms. Specifically, the two kinds of variogram matrix for the bivariate random field, $\boldsymbol{Z}(\boldsymbol{s})=\left\{Z_{1}(\boldsymbol{s}), Z_{2}(\boldsymbol{s})\right\}^{T}$, where $\boldsymbol{s} \in \mathbb{R}^{d}$, are:

$$
\gamma(\boldsymbol{h})=\left(\begin{array}{cc}
\gamma_{11}(\boldsymbol{h}) & \gamma_{12}(\boldsymbol{h}) \\
\gamma_{21}(\boldsymbol{h}) & \gamma_{22}(\boldsymbol{h})
\end{array}\right), \text { and } \boldsymbol{\nu}(\boldsymbol{h})=\left(\begin{array}{cc}
\nu_{11}(\boldsymbol{h}) & \nu_{12}(\boldsymbol{h}) \\
\nu_{21}(\boldsymbol{h}) & \nu_{22}(\boldsymbol{h})
\end{array}\right),
$$

where $\boldsymbol{h}=\boldsymbol{s}_{1}-\boldsymbol{s}_{2}$, and $\gamma_{11}(\boldsymbol{h})=\gamma_{22}(\boldsymbol{h})=\nu_{11}(\boldsymbol{h})=\nu_{22}(\boldsymbol{h}), \gamma_{12}(\boldsymbol{h})=$ $\gamma_{21}(\boldsymbol{h}), \nu_{12}(\boldsymbol{h})=\nu_{21}(\boldsymbol{h})$. In addition, the difference between $\gamma_{12}(\boldsymbol{h})\left(=\gamma_{21}(\boldsymbol{h})\right)$ and $\nu_{12}(\boldsymbol{h})\left(=\nu_{21}(\boldsymbol{h})\right)$ is a constant with respect to $\|\boldsymbol{h}\|$ for any combinations of parameters in the models.

Motivated by Genton et al. (2015a), we use visuanimation to dynamically display how the direct and cross variograms, as functions of $\|\boldsymbol{h}\|$, transit smoothly from boundedness to unboundedness by changing the parameters in the model. Movie 1 in the electronic supplementary material (if open with Adobe Acrobat Reader) shows visuanimations of the direct variograms, $\gamma_{11}(\boldsymbol{h})\left(=\gamma_{22}(\boldsymbol{h})=\nu_{11}(\boldsymbol{h})=\nu_{22}(\boldsymbol{h})\right)$, and the cross variograms, $\gamma_{12}(\boldsymbol{h})\left(=\gamma_{21}(\boldsymbol{h})\right)$ and $\nu_{12}(\boldsymbol{h})\left(=\nu_{21}(\boldsymbol{h})\right)$, from Models 1 and 3. Movie 2 shows the same from Models 2 and 4 . We fix some of the parameters, $\alpha_{1}=1, \alpha_{2}=2, \theta_{12}=$ $\theta_{21}=1$, and $\lambda=0.8$, and change the values of $\alpha$ and $\beta$ according to $\alpha \in$ $\{0.04,0.136, \ldots, 1.96\}$ and $\beta \in\{-1.96,-1.862, \ldots, 1.96\}$, which is the same setting as the one in Schlather and Moreva (2017). We also present some ex- 
ample plots here in Figure 1 and Figure 2. From the visuanimations and the figures, we see that the direct and cross variograms are bounded if and only if $\beta<0$ in Models 1 and 3 (Movie 1 and Figure 1), or if and only if $\beta_{1}<0$ and $\beta_{2}<0$ in Models 2 and 4 (Movie 2 and Figure 2). Because all of the entries are univariate variograms in the traditional variogram matrix (since $\lambda=0.8 \in[0,1])$ and in the pseudo variogram matrix, the direct and cross variograms from the four models should be less than quadratic growth, which can be seen from the visuanimations and the figures.
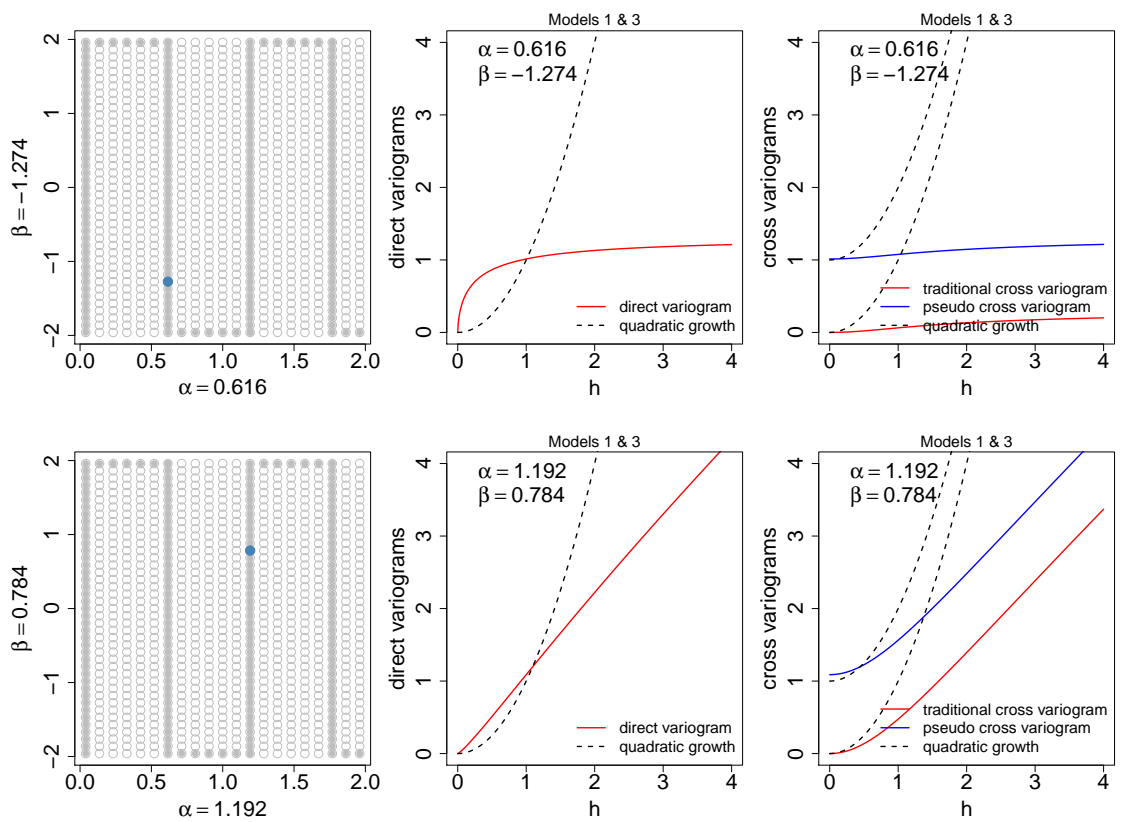

Fig. 1: Examples of direct variograms (center), cross variograms in the variogram matrix from Models 1 and 3 (right), and the corresponding parameter values (left).

\section{Variogram Matrix Models with Different Diagonal Entries}

One disadvantage of the models proposed in Sections 2 and 3 is that the diagonal entries in the variogram matrix are the same, that is, the variables $Z_{i}(s), i=1, \ldots, p$, all share the same direct variogram, which is too restrictive in practice. In this section, we propose a method of constructing parametric variogram matrices that possess different direct variograms and can 

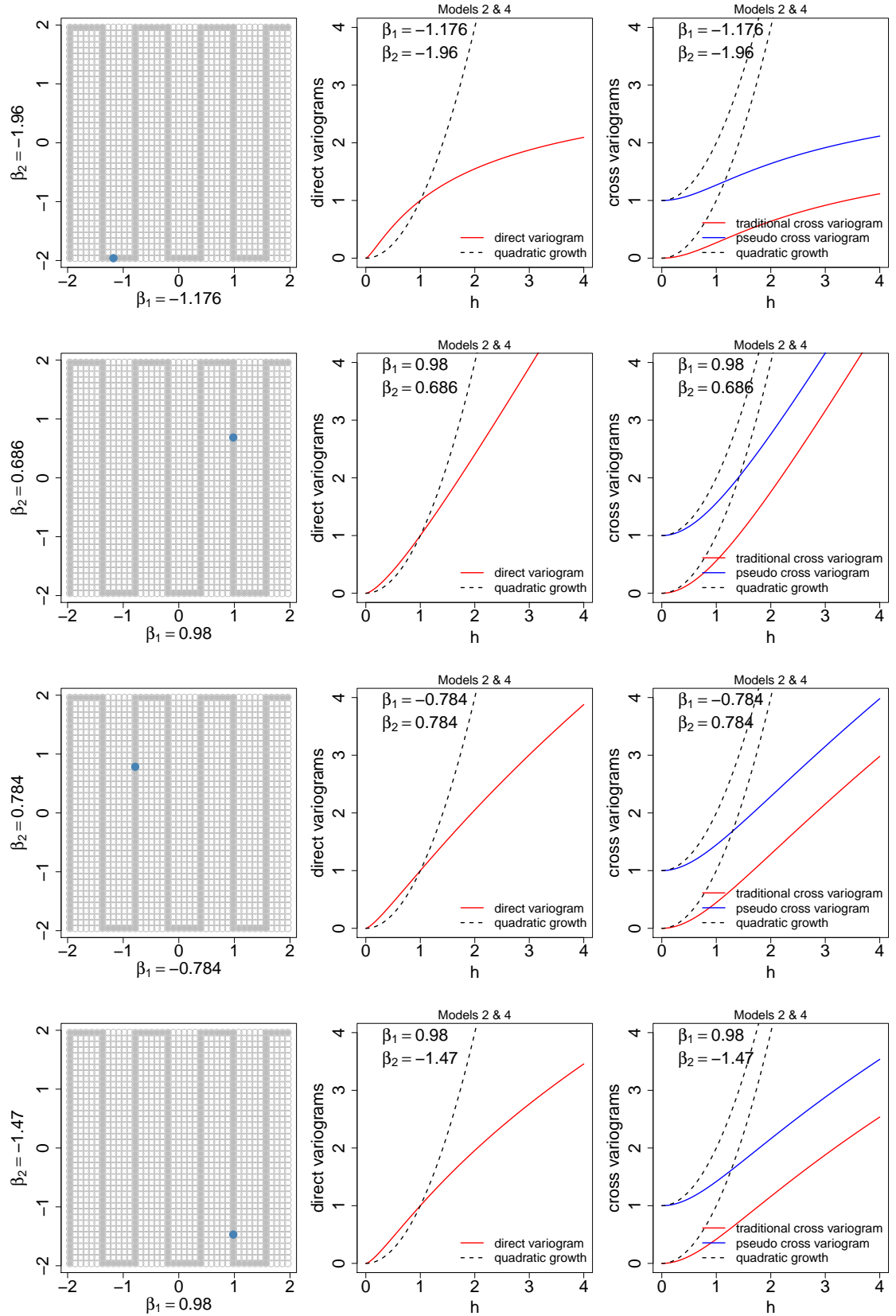

Fig. 2: Examples of direct variograms (center), cross variograms in the variogram matrix from Models 2 and 4 (right), and the corresponding parameter values (left). 
vary between boundedness and unboundedness based on the linear model of coregionalization (LMC) (Journel and Huijbregts 1978).

The LMC represents a vector random field as a linear combination of independent (or at least uncorrelated) univariate random fields. Specifically, we model the $p$-variate random field $\boldsymbol{Z}(\boldsymbol{s})=\left\{Z_{1}(s), \ldots, Z_{p}(s)\right\}^{T}, \boldsymbol{s} \in \mathbb{R}^{d}$, as

$$
Z_{i}(\boldsymbol{s})=\sum_{l=1}^{L} a_{i l} W_{l}(s), i=1, \ldots, p
$$

where $W_{l}(s), l=1, \ldots, L$ are independent univariate random fields defined on $\mathbb{R}^{d}$. Then, the entries in the traditional variogram matrix $\gamma(\boldsymbol{h})$ are given by

$$
\begin{aligned}
\gamma_{i j}(\boldsymbol{h}) & =\frac{1}{2} \operatorname{cov}\left\{Z_{i}(\boldsymbol{s}+\boldsymbol{h})-Z_{i}(\boldsymbol{s}), Z_{j}(\boldsymbol{s}+\boldsymbol{h})-Z_{j}(\boldsymbol{s})\right\} \\
& =\frac{1}{2} \operatorname{cov}\left[\sum_{l=1}^{L} a_{i l}\left\{W_{l}(\boldsymbol{s}+\boldsymbol{h})-W_{l}(\boldsymbol{s})\right\}, \sum_{l=1}^{L} a_{j l}\left\{W_{l}(\boldsymbol{s}+\boldsymbol{h})-W_{l}(\boldsymbol{s})\right\}\right] \\
& =\sum_{l=1}^{L} a_{i l} a_{j l} \frac{1}{2} \operatorname{var}\left\{W_{l}(\boldsymbol{s}+\boldsymbol{h})-W_{l}(\boldsymbol{s})\right\} \\
& =\sum_{l=1}^{L} a_{i l} a_{j l} \gamma_{l}(\boldsymbol{h}), \boldsymbol{s}, \boldsymbol{h} \in \mathbb{R}^{d}, i, j=1, \ldots, p
\end{aligned}
$$

where $\gamma_{l}(\boldsymbol{h}), l=1, \ldots, L$, is the univariate variogram of $W_{l}(\boldsymbol{s})$. The same results have been derived in Bourgault and Marcotte (1991). Hence, the diagonal entries of the variogram matrix $\gamma(\boldsymbol{h})$ are

$$
\gamma_{i i}(\boldsymbol{h})=\sum_{l=1}^{L} a_{i l}^{2} \gamma_{l}(\boldsymbol{h}), i, j=1, \ldots, p
$$

and can be varied by choosing different coefficients $a_{i l}, i=1, \ldots, p, l=$ $1, \ldots, L$.

By construction, a variogram matrix with the above entries is valid. If we choose the univariate variogram model (1) for $W_{l}(\boldsymbol{s}), l=1, \ldots, L$, that is, $\gamma_{\alpha_{l}, \beta_{l}}(\boldsymbol{h})$, where $0<\alpha_{l} \leq 2,-\infty<\beta_{l} \leq 2, l=1, \ldots, L$, then the direct and cross variograms, $\gamma_{i j}(\boldsymbol{h}), i, j=1, \ldots, p$, can vary between boundedness and unboundedness by altering the appropriate parameters $\beta_{l}, l=1, \ldots, L$ and the coefficients $a_{i l}, i=1, \ldots, p, l=1, \ldots, L$. To illustrate this, we look at the special case when $p=2$, that is, the bivariate case. Consider a joint intrinsically stationary bivariate random field $\boldsymbol{Z}(\boldsymbol{s})=\left\{Z_{1}(\boldsymbol{s}), Z_{2}(\boldsymbol{s})\right\}^{T}, \boldsymbol{s} \in \mathbb{R}^{d}$. 
Suppose that

$$
\begin{aligned}
& Z_{1}(s)=a_{11} W_{1}(s)+a_{12} W_{2}(s), \\
& Z_{2}(s)=a_{21} W_{1}(s)+a_{22} W_{3}(s),
\end{aligned}
$$

where $W_{1}(\boldsymbol{s}), W_{2}(\boldsymbol{s})$ and $W_{3}(\boldsymbol{s})$ are three independent intrinsically stationary univariate random fields defined on $\mathbb{R}^{d}$. The traditional variogram matrix of the bivariate random field, $\boldsymbol{Z}(\boldsymbol{s})=\left\{Z_{1}(\boldsymbol{s}), Z_{2}(\boldsymbol{s})\right\}^{T}, \boldsymbol{s} \in \mathbb{R}^{d}$, is given by

$$
\gamma(\boldsymbol{h})=\left[\begin{array}{cc}
a_{11}^{2} \gamma_{1}(\boldsymbol{h})+a_{12}^{2} \gamma_{2}(\boldsymbol{h}) & a_{11} a_{21} \gamma_{1}(\boldsymbol{h}) \\
a_{21} a_{11} \gamma_{1}(\boldsymbol{h}) & a_{21}^{2} \gamma_{1}(\boldsymbol{h})+a_{22}^{2} \gamma_{3}(\boldsymbol{h})
\end{array}\right]
$$

where $\boldsymbol{h}=\boldsymbol{s}_{1}-\boldsymbol{s}_{2}$, and $\gamma_{1}(\boldsymbol{h}), \gamma_{2}(\boldsymbol{h})$ and $\gamma_{3}(\boldsymbol{h})$ are the univariate variograms of $W_{1}(\boldsymbol{s}), W_{2}(\boldsymbol{s})$ and $W_{3}(\boldsymbol{s})$, respectively.

Then, we suppose that $\gamma_{1}(\boldsymbol{h}), \gamma_{2}(\boldsymbol{h})$ and $\gamma_{3}(\boldsymbol{h})$ are of the form (1), and hence can vary between boundedness and unboundedness. If $\gamma_{1}, \gamma_{2}$ and $\gamma_{3}$ are all bounded (i.e., $\beta_{1}<0, \beta_{2}<0$ and $\beta_{3}<0$ ), then all the direct and cross variograms are bounded; similarly, if $\gamma_{1}, \gamma_{2}$ and $\gamma_{3}$ are all unbounded (i.e., $\beta_{1} \geq 0, \beta_{2} \geq 0$ and $\beta_{3} \geq 0$ ), then all the direct and cross variograms are unbounded. In two cases, the cross variograms are bounded and one of the direct variograms is unbounded: when $\gamma_{1}$ and $\gamma_{2}$ are bounded, $\gamma_{3}$ is unbounded, and $a_{22} \neq 0$; and when $\gamma_{1}$ and $\gamma_{3}$ are bounded, $\gamma_{2}$ is unbounded, and $a_{12} \neq$ 0 . On the other hand, the direct variograms are unbounded and the cross variograms are bounded when $\gamma_{1}$ is bounded, $\gamma_{2}$ and $\gamma_{3}$ are unbounded, and $a_{12} \neq 0, a_{22} \neq 0$.

Therefore, this class of LMC-based bivariate variogram matrix incorporates the following four situations:

$$
\left[\begin{array}{ll}
\mathrm{B} & \mathrm{B} \\
\mathrm{B} & \mathrm{B}
\end{array}\right],\left[\begin{array}{ll}
\mathrm{U} & \mathrm{U} \\
\mathrm{U} & \mathrm{U}
\end{array}\right],\left[\begin{array}{ll}
\mathrm{B} & \mathrm{B} \\
\mathrm{B} & \mathrm{U}
\end{array}\right],\left[\begin{array}{ll}
\mathrm{U} & \mathrm{B} \\
\mathrm{B} & \mathrm{U}
\end{array}\right],
$$

where "B" stands for a bounded entry and "U" for an unbounded entry.

Recall that according to (3), the following situation cannot exist:

$$
\left[\begin{array}{ll}
B & U \\
\text { U } & B
\end{array}\right] \text {. }
$$

Therefore, the only case that is not covered by our model is

$$
\left[\begin{array}{ll}
B & U \\
U & U
\end{array}\right] \text {. }
$$

Although this class of variogram matrix does not cover all the cases where direct and cross variograms can vary between boundedness and unboundedness, it is more flexible than Models 1 or 3 in the sense that the diagonal 
entries in the variogram matrix can be different. Moreover, the direct variograms can have different growth rate, which are controlled by the parameters $\beta_{l}, l=1, \ldots, L$ and the coefficients $a_{i l}, i=1, \ldots, p, l=1, \ldots, L$.

Unfortunately, this LMC-based approach cannot be used to construct a pseudo variogram matrix with different diagonal entries. Under the assumption of (7), the entries in the pseudo variogram matrix $\boldsymbol{\nu}(\boldsymbol{h})$ are

$$
\begin{aligned}
\nu_{i j}(\boldsymbol{h}) & =\frac{1}{2} \operatorname{var}\left\{Z_{i}(\boldsymbol{s}+\boldsymbol{h})-Z_{j}(\boldsymbol{s})\right\} \\
& =\frac{1}{2} \operatorname{var}\left\{\sum_{l=1}^{L} a_{i l} W_{l}(\boldsymbol{s}+\boldsymbol{h})-\sum_{l=1}^{L} a_{j l} W_{l}(\boldsymbol{s})\right\} \\
& =\sum_{l=1}^{L} \frac{1}{2} \operatorname{var}\left\{a_{i l} W_{l}(\boldsymbol{s}+\boldsymbol{h})-a_{j l} W_{l}(\boldsymbol{s})\right\}, \quad \boldsymbol{s}, \boldsymbol{h} \in \mathbb{R}^{d}, i, j=1, \ldots, p,
\end{aligned}
$$

which cannot be expressed as functions of the univariate variograms $\gamma_{l}(\boldsymbol{h})$ for $W_{l} \mathrm{~s}$.

\section{Discussion}

In this paper, we proposed several parametric models for both the traditional and pseudo variogram matrices that can span from joint second-order to intrinsic stationarity of a vector random field. Our models generalized the univariate variogram model in Schlather and Moreva (2017) to variogram matrices for vector random fields, which preserved the flexibility of varying between boundedness and unboundedness for the entries of the variogram matrix. In addition, compared to other related similar work such as Ma (2011a), we provided a simple and flexible class of parametric variogram matrices, where the direct variograms in the diagonal entries and cross variograms in the off-diagonal entries can vary between boundedness and unboundedness by changing the values of parameters in the model. Based on the method of latent dimension for constructing variogram matrices in Ma (2011a), each entry of our variogram matrix model is a linear combination of univariate variograms on a higher dimension, each of these univariate variograms is a linear combination of Bernstein functions, and each of the Bernstein functions is chosen as the univariate variogram model in Schlather and Moreva (2017).

The variogram matrix model based on the method of latent dimensions has a restriction that all the variables share the same direct variogram. Hence, we proposed a procedure based on the LMC for constructing a traditional 
variogram matrix that has different direct variograms with different degrees of boundedness and unboundedness, by assigning the parameters in the univariate variogram models and the coefficients in the linear combinations. This class of variogram matrix, however, does not cover all the cases where the direct and cross variograms can vary between boundedness and unboundedness, and one case is thus left as an open problem. An example of this case (i.e., the [B U;U U] case) is presented in Arroyo and Emery (2017), and Maleki and Emery (2017) presented a similar model corresponding to the [B B;B U] case. Moreover, the LMC approach is not feasible for constructing pseudo variogram matrices with different diagonal entries.

In Section 4, we presented a visuanimation that illustrated our model allowing for bounded and unbounded direct and cross variograms in the variogram matrix in the bivariate case. In future work, it would be interesting to simulate a bivariate random field and its corresponding direct and cross variograms. Another use of such simulations where we can transit smoothly from second-order to intrinsic stationarity is to then evaluate methods developed under second-order stationarity and see how they behave under intrinsic stationarity. However, simulation of a vector random field is difficult and time consuming when the field is intrinsically stationary but not second-order stationary, because the direct and cross variograms might be unbounded and the cross-covariance matrix is not well defined. Several exact and approximate simulation methods have been proposed, for example, circulant embedding (Stein 2002; Danudirdjo and Hirose 2011), spectral representations (Arroyo and Emery 2017) and turning bands (Emery 2008). More recently, Moreva and Schlather (2018) proposed extensions of the cut-off circulant embedding approach for fast and exact simulation of univariate and bivariate Gaussian random fields. The readers are referred to Chilès and Delfiner (2012), Lantuéjoul (2002), Arroyo and Emery (2017) and the references therein for more details. Another direction for study would be to construct a pseudo variogram matrix with different diagonal entries.

The models we developed in this work could be applied for meteorological problems, such as in Schlather and Tawn (2003). Specifically, the volume of rainfall and its spatial spread determine the degree of flooding at sites. Different types of rainfall (such as local storms and cyclonic rainfall) can occur at different locations or different time of the year at one location. As discussed in Section 1, it is advantageous to use a model that incorporates both bounded and unbounded variograms when the underlying causative process is unclear. Our variogram matrix models for vector random fields could be then used to 
model multiple rainfall events in a year over a spatial domain, and from the parameter estimates we could infer the types of rainfall.

Acknowledgements The authors are grateful to Martin Schlather for providing the $\mathrm{R}$ code used in Schlather and Moreva (2017), based on which the visuanimations of direct and cross variograms in Movies 1 and 2 in the electronic supplementary material were produced.

\section{References}

Apanasovich TV, Genton MG (2010) Cross-covariance functions for multivariate random fields based on latent dimensions. Biometrika 97(1):15-30

Arroyo D, Emery X (2017) Spectral simulation of vector random fields with stationary gaussian increments in d-dimensional euclidean spaces. Stochastic Environmental Research and Risk Assessment 31(7):1583-1592

Bochner S (2005) Harmonic analysis and the theory of probability. Courier Corporation

Bourgault G, Marcotte D (1991) Multivariable variogram and its application to the linear model of coregionalization. Mathematical Geology 23(7):899 928

Brown BM, Resnick SI (1977) Extreme values of independent stochastic processes. Journal of Applied Probability 14(4):732-739

Chilès JP, Delfiner P (2012) Geostatistics: Modeling Spatial Uncertainty, 2nd edn. John Wiley \& Sons

Clark I, Basinger K, Harper W (1989) MUCK-A Novel Approach to CoKriging. In: B. E. Buxton (Ed.), Proceedings of the Conference on Geostatistical, Sensitivity, and Uncertainty: Methods for Ground-Water Flow and Radionuclide Transport Modeling, Batelle Press, Columnbus, pp 473-494

Cressie N, Wikle CK (1998) The variance-based cross-variogram: you can add apples and oranges. Mathematical Geology 30(7):789-799

Cressie NA (1991) Statistics for Spatial Data: Wiley Series in Probability and Mathematical Statistics. John Wiley \& Sons

Danudirdjo D, Hirose A (2011) Synthesis of two-dimensional fractional Brownian motion via circulant embedding. In: 18th IEEE International Conference on Image Processing, pp 1085-1088

Davison AC, Gholamrezaee MM (2012) Geostatistics of extremes. Proceedings of the Royal Society A 468(2138):581-608

Emery X (2008) A turning bands program for conditional co-simulation of cross-correlated Gaussian random fields. Computers \& Geosciences $34(12): 1850-1862$ 
Genton MG, Kleiber W (2015) Cross-covariance functions for multivariate geostatistics. Statistical Science 30(2):147-163

Genton MG, Castruccio S, Crippa P, Dutta S, Huser R, Sun Y, Vettori S (2015a) Visuanimation in statistics. Stat 4(1):81-96

Genton MG, Padoan SA, Sang H (2015b) Multivariate max-stable spatial processes. Biometrika 102(1):215-230

Huang C, Yao Y, Cressie N, Hsing T (2009) Multivariate intrinsic random functions for cokriging. Mathematical Geosciences 41(8):887-904

Journel AG, Huijbregts CJ (1978) Mining Geostatistics. Academic Press, San Diego

Kabluchko Z, Schlather M (2010) Ergodic properties of max-infinitely divisible processes. Stochastic Processes and their Applications 120(3):281-295

Kabluchko Z, Schlather M, De Haan L (2009) Stationary max-stable fields associated to negative definite functions. The Annals of Probability $37(5): 2042-2065$

Lantuéjoul C (2002) Geostatistical Simulation: Models and Algorithms. Springer Science \& Business Media

Ma C (2005) Linear combinations of space-time covariance functions and variograms. IEEE Transactions on Signal Processing 53(3):857-864

Ma C (2009) Intrinsically stationary variograms in space and time. Theory of Probability \& Its Applications 53(1):145-155

Ma C (2011a) A class of variogram matrices for vector random fields in space and/or time. Mathematical Geosciences 43(2):229-242

Ma C (2011b) Vector random fields with second-order moments or secondorder increments. Stochastic Analysis and Applications 29(2):197-215

Maleki M, Emery X (2017) Joint simulation of stationary grade and nonstationary rock type for quantifying geological uncertainty in a copper deposit. Computers \& Geosciences 109:258-267

Matheron G (1963) Principles of geostatistics. Economic Geology 58(8):12461266

Matheron G (1965) Les variables régionalisées et leur estimation: une application de la théorie des fonctions aléatoires aux sciences de la nature. Masson et CIE

Molchanov I, Stucki K (2013) Stationarity of multivariate particle systems. Stochastic Processes and their Applications 123(6):2272-2285

Moreva O, Schlather M (2018) Fast and exact simulation of univariate and bivariate Gaussian random fields. Stat 7(1):e188 
Myers DE (1982) Matrix formulation of co-kriging. Mathematical Geology $14(3): 249-257$

Myers DE (1991) Pseudo-cross variograms, positive-definiteness, and cokriging. Mathematical Geology 23(6):805-816

Myers DE (1992) Kriging, cokriging, radial basis functions and the role of positive definiteness. Computers \& Mathematics with Applications 24(12):139 148

Porcu E, Schilling RL (2011) From Schoenberg to Pick-Nevanlinna: Toward a complete picture of the variogram class. Bernoulli 17(1):441-455

Porcu E, Mateu J, Zini A, Pini R (2007) Modelling spatio-temporal data: a new variogram and covariance structure proposal. Statistics \& Probability Letters $77(1): 83-89$

Schlather M, Moreva O (2017) A parametric model bridging between bounded and unbounded variograms. Stat 6(1):47-52

Schlather M, Tawn JA (2003) A dependence measure for multivariate and spatial extreme values: Properties and inference. Biometrika 90(1):139-156

Schoenberg IJ (1938) Metric spaces and completely monotone functions. Annals of Mathematics pp 811-841

Stein ML (2002) Fast and exact simulation of fractional Brownian surfaces. Journal of Computational and Graphical Statistics 11(3):587-599

Thibaud E, Mutzner R, Davison AC (2013) Threshold modeling of extreme spatial rainfall. Water Resources Research 49(8):4633-4644

Wackernagel H (1988) Geostatistical techniques for interpreting multivariate spatial information. In: Quantitative Analysis of Mineral and Energy Resources, Springer, pp 393-409

Wackernagel H (2003) Multivariate Geostatistics: An Introduction with Applications, 3rd edn. Springer Science \& Business Media

Wadsworth JL, Tawn JA (2013) Efficient inference for spatial extreme value processes associated to log-Gaussian random functions. Biometrika 101(1):1-15

Wang Y, Stoev SA (2010) On the structure and representations of max-stable processes. Advances in Applied Probability 42(3):855-877 